\title{
COVID-19, Telemedicine, and Patient Empowerment in HIV Care and Research
}

\author{
Ofole Mgbako ${ }^{1,2} \cdot$ Emily H. Miller $^{1} \cdot$ Anthony F. Santoro $^{2} \cdot$ Robert H. Remien $^{2} \cdot$ Noga Shalev $^{1} \cdot$ Susan Olender ${ }^{1}$. \\ Peter Gordon ${ }^{1} \cdot$ Magda E. Sobieszczyk ${ }^{1}$
}

Published online: 21 May 2020

(c) Springer Science+Business Media, LLC, part of Springer Nature 2020

Beyond the tragic loss of life, the COVID-19 pandemic has disrupted HIV care delivery throughout the U.S. To meet the needs of people living with HIV/AIDS (PLWHA) along the care continuum, health systems have expanded the use of telemedicine (e.g., video, telephone visits) to maintain HIV treatment adherence and care engagement. Despite some promising early results $[1,2]$, providers must consider the implications of telemedicine on the patient-provider relationship and the establishment of trust in ongoing care, particularly for the most vulnerable. An approach focused on patients' values and preferences provides an opportunity to empower PLWHA in this new paradigm of HIV care. As HIV primary care physicians and researchers working in New York City, we have grappled with the benefits and challenges of telemedicine as we seek continuity in our work during the COVID-19 pandemic.

The move toward telemedicine in HIV care has increased over the past decade [2,3]. A recent study of 371 PLWHA found $57 \%$ of respondents were more likely to use telemedicine for their HIV care compared to in-person [4]. This sample was predominantly US-born, with a higher education level and perceived HIV-related stigma [4]. Broad acceptance of telemedicine may be true only in specific subgroups of PLHWA. Due to the notable health disparities in both the COVID-19 and HIV-1 pandemics, we believe it is important to work towards equitable models of HIV care [5]. In this paper, we present a patient case from our HIV clinic and

Ofole Mgbako

om2292@cumc.columbia.edu

1 Division of Infectious Disease, Department of Internal Medicine, Columbia University Irving Medical Center, New York, NY, USA

2 HIV Center for Clinical and Behavioral Studies, NY State Psychiatric Institute and Columbia University, New York, NY, USA explore the potential tradeoffs between patient-centered care and telemedicine.

\section{View from the Clinic}

A young African American woman with uncontrolled HIV and multiple comorbidities presented for a follow-up video visit appointment. The patient was unemployed and fearful of leaving her apartment due to risk of COVID-19 given her compromised immune status. During the visit, the patient seemed stressed by multiple difficulties that arose with the video visit interface, including many connectivity disruptions; as a result, her appointment was converted to a telephone visit. During the call she reported possible side effects from her complicated antiretroviral treatment (ART) regimen. She also required specialty care for her other medical conditions. Her provider scheduled an in-person visit in one month.

From the provider perspective, the patient had a complex HIV history and low health literacy, factors often associated with poor HIV-related outcomes. Balancing the need for routine bloodwork and supportive in-person services with the patient's reluctance to leave her apartment was challenging. The patient also seemed overwhelmed by the technology such that the telemedicine visit negatively influenced the provider's ability to build rapport.

\section{A Patient-Centered Approach for PLWHA: Communication, Trust and the Human Touch}

Despite COVID-19, clinicians must have a deep understanding of the most important aspects of the patient-provider relationship vital for positive outcomes along the HIV care continuum. Studies have found that high quality communication from providers (e.g. active listening, clear explanation) 
and a strong patient-provider relationship leads to improved patient engagement in HIV care and better ART adherence $[6,7]$. These findings, however, are based on in-person visits, which allow the patient to sit face-to-face with their provider, discuss sensitive medical issues, and engage in shared decision-making [8]. More research is necessary to establish which elements of communication and the patientprovider relationship are vital on a telemedicine platform. Furthermore, providers must learn to proficiently use new technology, while balancing quality communication, writing clinical documentation, ordering laboratory testing and building rapport.

Trust is another essential component of HIV care for the most disproportionately affected PLWHA. Racial/ethnic and sexual minorities living with HIV have consistently reported mistrust of providers and the medical system, often due to stigmatizing healthcare experiences and the history of mistreatment from the medical establishment [9-12]. Actively seeking feedback on telemedicine from PLWHA from these communities establishes an HIV care delivery system that incorporates their concerns. Overcoming generations of mistrust must remain a central goal in a new telemedicine era of HIV care, which is accelerating as a result of COVID-19.

Notably, the move towards telemedicine in HIV care precludes the physical exam, which many providers believe forges connection and builds trust. Dr. Abraham Verghese, a physician who emphasizes the importance of the physical exam from treating patients at the height of the AIDS epidemic, writes, "In these modern times, when medical care is so fractured, a thorough exam conveys attentiveness in addition to providing comfort and reassurance. At the end of this ritual, physician and patient are no longer strangers but are bonded through touch [13]." It is unclear whether the same trust and connection important for HIV care outcomes can be maintained under a telemedicine paradigm free of consistent human touch due to COVID-19. However, technological advancements in HIV care are reinventing how providers and patients define connection. This provides an opportunity to identify best practices, informed by the perspectives of PLWHA with complex histories and from historically marginalized groups, and provide training to providers at all levels.

\section{Telemedicine Challenges in HIV Clinical Care Delivery and the Adopt IT Model}

During the COVID-19 pandemic, some PLWHA may prefer virtual visits. Advantages include convenience, decreased travel time/expenses, avoiding stigmatizing clinic experiences, and minimization of infectious risk/social contact. However, the rollout of telemedicine has not been without challenges. In one recent study, providers reported difficulties establishing rapport and an emotional connection with patients during telemedicine visits [14]. Another study showed patients felt rushed, less involved, and less comfortable asking sensitive questions due to privacy concerns [15]. Furthermore, care for PLWHA is typically performed by a team of doctors, nurses, mental health providers, social workers, and care coordinators. An approach to team-based care will need to be incorporated into the telemedicine model.

The expansion of telemedicine into HIV care has the potential to worsen disparities in access for those with certain social disadvantages, such as low health literacy or those without internet or adequate phone or computerbased technology [16]. In 2018, our site's Adopt IT program used individualized coaching for PLWHA with low health literacy to increase patient capacity to utilize their personal electronic health record [17]. We focused on overcoming gaps in knowledge, experience and comfort to ensure these patients had opportunities and confidence to use such tools. During COVID-19, our care coordinators have been proactively contacting patients to check on medication supply, food and housing security, and to help set up technology during the pandemic. And we have preserved options for in-person visits with appropriate safety measures especially for those with newly diagnosed HIV, new to the clinic, nonEnglish-speaking, with limited access to technology, or low health or technology literacy. This approach can be tailored to the recent expansion of telemedicine.

\section{Telemedicine Challenges in HIV Research}

Technology has been increasingly incorporated into research across the HIV care continuum, with particular success engaging young PLWHA $[18,19]$. Amidst the COVID19 pandemic, HIV researchers have been forced to adopt technology-based methods to continue their work. However, technology's advantages may come with drawbacks, such as lack of participant-researcher rapport and less confidentiality, especially with qualitative research [20]. Furthermore, the rapid adoption of telemedicine in HIV care carries risk for HIV research that relies on clinic-based recruitment. Telemedicine's potential negative impact on patient-provider rapport could adversely impact patients' openness to learning about opportunities to participate in research. Technology-based research may also create significant barriers for accurate reporting in studies in which participant trust is essential (e.g. disclosure of sexual behaviors or trauma). Lastly, for studies with longitudinal design, maintaining positive participant-researcher relationships is critical to successfully retain participants over time.

As in HIV care, the participant-researcher connection is critical for recruiting and retaining PLWHA from 
marginalized and hard-to-reach communities. The possible weakening of the participant-researcher connection in HIV research risks introducing sampling bias and limiting the generalizability of findings to PLWHA who are younger, more tech-savvy, and from higher socioeconomic backgrounds, further exacerbating disparities in HIV research $[4,15,21-23]$. Researchers must balance technological innovation and the participant-researcher connection in order to engage the most vulnerable communities of PLWHA. This requires adaptable recruitment and engagement strategies, providing participants with choices (e.g., Zoom, telephone), and working collaboratively with participants to identify and overcome barriers to research participation.

\section{Conclusion}

In the time of COVID-19, telemedicine in HIV care must first serve the needs of the patient. The necessary growth of telemedicine during a global pandemic must not come at the cost of a patient-centered approach to HIV care, rooted in trust, patient-provider connection, and effective communication. This moment provides a unique opportunity to build a telemedicine model in HIV care that empowers patients. HIV providers should continually appraise their telemedicine programs through patient feedback, focus on increasing access among the most at-risk PLWHA, and consider provider education training on optimal communication to enhance trust and connection. HIV providers and researchers also should provide PLWHA with flexible options that meet their needs, address knowledge/training gaps and improve our HIV care systems. PLWHA with higher medical complexity and who are socially vulnerable - as in our patient case - will continue to be the ones lost to care and excluded from research if the telemedicine system is not designed with them in mind.

\section{References}

1. Rogers BG, Coats CS, Adams E, et al. Development of telemedicine infrastructure at an LGBTQ+ clinic to support HIV prevention and care in response to COVID, Providence RI. AIDS Behav. 2020. https://doi.org/10.1007/s10461-020-02895-1.

2. Dandachi D, Freytag J, Giordano TP, Dang BN. It is time to include telehealth in our measure of patient retention in HIV care. AIDS Behav. 2020. https://doi.org/10.1007/s10461-020-02880-8.

3. Brantley AD, Page KM, Zack B, et al. Making the connection: using videoconferencing to increase linkage to care for incarcerated persons living with HIV post-release. AIDS Behav. 2019;23(Suppl 1):32-40. https://doi.org/10.1007/s1046 1-018-2115-4

4. Dandachi D, Dang BN, Lucari B, Teti M, Giordano TP. Exploring the attitude of patients with HIV about using telehealth for HIV care. AIDS Patient Care STDS. 2020;34(4):166-72. https://doi. org/10.1089/apc.2019.0261.
5. "COVID-19 Racial Disparities in U.S. Counties," AmfAR; May 2020. ehe.amfar.org/disparities

6. Flickinger TE, Saha S, Moore RD, Beach MC. Higher quality communication and relationships are associated with improved patient engagement in HIV care. J Acquir Immune Defic Syndr. 2013;63(3):362-6. https://doi.org/10.1097/QAI.0b013e3182 95b86a.

7. Dang BN, Westbrook RA, Black WC, Rodriguez-Barradas MC, Giordano TP. Examining the link between patient satisfaction and adherence to HIV care: a structural equation model. PLoS ONE. 2013;8(1):e54729. https://doi.org/10.1371/journ al.pone. 0054729 .

8. McCoy L. HIV-positive patients and the doctor-patient relationship: perspectives from the margins. Qual Health Res. 2005;15(6):791-806.

9. Pellowski JA, Price DM, Allen AM, Eaton LA, Kalichman SC, The differences between medical trust and mistrust and their respective influences on medication beliefs and ART adherence among African-Americans living with HIV. Psychol Health. 2017;32(9):1127-39. https://doi.org/10.1080/08870 446.2017.1324969.

10. Freeman R, Gwadz MV, Silverman E, et al. Critical race theory as a tool for understanding poor engagement along the HIV care continuum among African American/Black and Hispanic persons living with HIV in the United States: a qualitative exploration. Int J Equity Health. 2017;16:54.

11. Eaton LA, Driffin DD, Kegler C, et al. The role of stigma and medical mistrust in the routine health care engagement of black men who have sex with men. Am J Public Health. 2015;105(2):e75e82. https://doi.org/10.2105/AJPH.2014.302322.

12. Washington HA, Apartheid M. The dark history of medical experimentations Black Americans from colonial times to the present. New York: Doubleday; 2006.

13. Verghese A. A touch of sense. Health Aff. 2009;28(4):1177-82.

14. Anderson K, Francis T, Ibanez-Carrasco F, Globerman J. Physician's perceptions of telemedicine in HIV care provision: a cross-sectional web-based survey. JMIR Public Heal Surveill. 2017;3(2):e31.

15. Gordon HS, Solanki P, Bokhour BG, Gopal RK. "I'm not feeling like i'm part of the conversation" patients' perspectives on communicating in clinical video telehealth visits. J Gen Intern Med. 2020. https://doi.org/10.1007/s11606-020-05673-w.

16. Pellowski JA, Kalichman SC, Matthews KA, Adler N. A pandemic of the poor: Social disadvantage and the US HIV epidemic. Am Psychol. 2013;68(4):197-209.

17. Kabemba F, Davila MG, Olender S. myNYP Adopt IT: A coaching intervention to improve uptake of a personal health record (PHR). 2019 Ryan White HIV/AIDS Clinical Conference; 2019, pp. 4-7; New Orleans, LA.

18. Muessig KE, Nekkanti M, Bauermeister J, Bull S, Hightow-Weidman LB. A systematic review of recent smartphone, internet and Web 20 interventions to address the HIV continuum of care. Curr HIV/AIDS Rep. 2015;12(1):173-90.

19. Hightow-Weidman LB, Muessig KE, Bauermeister J, Zhang C, LeGrand S. Youth, technology, and HIV: recent advances and future directions. Curr HIV/AIDS Rep. 2015;12(4):500-15.

20. Hubach RD, O'Neil A, Stowe M, Giano Z, Curtis B, Fisher CB. Perceived confidentiality risks of mobile technology-based ecologic momentary assessment to assess high-risk behaviors among rural men who have sex with men. Arch Sex Behav. 2020. https:// doi.org/10.1007/s10508-019-01612-x.

21. Shacham E, Stamm K, Overton ET. Can you hear me now? Limited use of technology among an urban HIV-infected cohort. AIDS Care. 2009;21(8):1000-6.

22. Menezes P, Eron J, Leone P, Adimora A, Wohl D, Miller W. Recruitment of HIV/AIDS treatment-naïve patients to clinical 
trials in the HAART era: influence of gender, sexual orientation and race. HIV Med. 2011;12(3):183-91.

23. Batista P, Deren S, Banfield A, Silva E, Cruz M, Garnes P, et al. Challenges in recruiting people who use drugs for HIV-related biomedical research: perspectives from the field. AIDS Patient Care. STDs. 2016;30(8):379-84.
Publisher's Note Springer Nature remains neutral with regard to jurisdictional claims in published maps and institutional affiliations. 